\title{
Gestión de Procesos de Negocios en el Sector Público
}

\section{Business Process Management in the Public Sector}

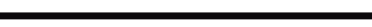 \\ INFORMACIÓN DEL ARTÍCULO \\ Fecha de recepción: 9 de febrero de 2019. \\ Fecha de aceptación: 13 de junio de 2019}

1 PhD en Investigación Operativa, Atlantic University. Docente e investigador de la Universidad del Salvador-Argentina.

E-mail: sergio.salimbeni@usal.edu.ar Código ORCID:

https://orcid.org/0000-0002-7365-1053

CITACIÓN: Salimbeni, S. (2019). Gestión de Procesos de Negocios en el Sector Público. Podium, 35, 69-86. doi:10.31095/podium.2019.35.5

ENLACE DOI:

http://dx.doi.org/10.31095/podium.201 9.35 .5

\section{Resumen}

Este artículo tiene por objeto hallar las diferencias de la gestión por procesos entre entornos públicos y privados, y sus factores clave de éxito para la implementación en la administración gubernamental. Esta investigación de enfoque mixto y desde la perspectiva de las ciencias económicas y empresariales, consta de dos etapas: una cualitativa y otra cuantitativa. Al término de la primera fase se evidenciaron diferencias en cuanto a la priorización de factores políticos por sobre los del interés público, la falta de consciencia en la optimización de recursos, el sobredimensionamiento de la estructura, el solapamiento de tareas y la falta de capacitación del personal. Como factores clave para su implementación en la administración gubernamental se validan un fuerte patrocinio, capacitación adecuada y disponibilidad de personal interno con experiencia en en la materia.

\section{Palabras Clave:}

Sector Público, Gobierno Electrónico, Gestión de Procesos de Gobierno, Gestión de Procesos de Negocios, Mejoras de Procesos de Negocios, Sistemas de Simulación.

Clasificación JEL: L3, L33.

\begin{abstract}
This article purports to ascertain the management process differences that occur between public and private surroundings and to show key success factors for implementing government administration. This mixed focus research is presented from the perspective of economic and business sciences; it consists of two stages: one is qualitative, while the other is quantitative. Upon completion of the first phase, it will become evident that political factors are often priorized over public interest. There is a lack of consciousness in the optimization of resources; structures are over dimensioned, the fact that tasks are not complied with, is covered up and the personnel is not sufficiently skilled. Key factors for implementation of government of administration validate a strong sponsorship and adequate training and availability of internal personnel that are experienced in the subject.
\end{abstract}

\section{Keywords:}

Public sector, electronic government, management of government processes, management of business processes, improvement of business processes, simulation system.

JEL Classification: L3, L33.

\section{9}

PODIUM No. 35, Junio 2019, pp. 69-86

Universidad Espíritu Santo - UEES

ISSN: 1390-5473 e-ISSN: 2588-0969 


\section{Introducción}

El enfoque de Gestión por Procesos, la Inteligencia de Procesos y las nuevas herramientas de simulación, pueden colaborar con la Gestión de Procesos en Gobiernos, también denominada GPM (Government Process Management). Teniendo en cuenta que la mayoría de las herramientas y conceptos de gestión estratégica se han desarrollado en el sector privado, cabe conocer si es válido aplicarlos al sector público, o cómo podrían adaptarse teniendo en cuenta sus realidades actuales. Alford (2001) planteaba ya en sus trabajos de Gestión Estratégica si las diferencias entre ambos sectores era una barrera para el uso de las mismas en ámbitos gubernamentales. Se explora también la importancia del GPM en el gobierno electrónico y las diferencias clave halladas entre el sector público y el privado desde la perspectiva de procesos, tanto en la Argentina como en otros países. Hay una gran cantidad de estudios científicos sobre los beneficios de la Gestión de Procesos de Negocios (BPM - Business Process Management), la mayoría de ellos enfocados en la actividad privada, por lo tanto, se considera necesario profundizar la aplicación del BPM al sector público.

En diferentes estudios desarrollados en países de todo el mundo se están evaluando las diferencias existentes entre la Gestión por Procesos en el sector privado y el público, como así también estudiando herramientas que permitan adaptar y aplicar la Gestión por Procesos a la Administración Pública.
Con idiosincrasias bien diferentes, desde Alemania, Australia, Estonia y Suecia pasando por Brasil, Perú y Ecuador, existen factores $y$ patrones de comportamiento similares, los cuales dificultan la implementación del BPM en el sector público. El modelo de Organización y Gestión por Procesos es una herramienta poderosa para mejorar el desempeño en la Gestión Pública; esto es debido a la virtud de la Gestión por Procesos de estar orientada a generar los productos finales primarios ajustados a las necesidades y expectativas de los clientes/ciudadanos (Marchán y Oviedo, 2011).

Existe un espectro muy amplio de conceptos y herramientas de gestión estratégica, sin embargo, hay un marco de referencia esencial en el campo de la Gestión Pública y la Gestión Estratégica: la "Política Modelo para los Negocios" de la Escuela de Negocios de Harvard (Bower, Bartlett, Christensen, Pearson, y Andrews, 1991). Conforme esta, la Nueva Gestión Pública engloba tres factores a ser considerados en el campo de la Gestión Estratégica: 1) qué productos/servicios ofrecer a qué mercados (la creación de valor), 2) el ambiente, y 3) las capacidades organizacionales.

Dado que la mayoría de los conceptos y herramientas de la Gestión Estratégica fueron desarrollados en el sector privado, cabe preguntarse: ¿pueden ser aplicados al sector público? Esta solía ser la pregunta inicial del capítulo de la Gestión Estratégica años atrás (Alford, 2001). La Estrategia Corporativa se originó en los 
Estados Unidos en la década de 1960, luego se difundió hacia Gran Bretaña, Australia, Nueva Zelanda y el resto de Europa del Norte y a partir de allí se ha estado adaptando globalmente (Alford y Greve, 2017). Los gobiernos eran percibidos, y lo son aún en varios países, como sobredimensionados e ineficientes; este concepto está reforzado también por la creencia generalizada de que el sector privado es más eficiente que el público. En cierto modo, ello se justifica basándose en la observación de las herramientas informáticas disponibles en uno y otro sector y la cantidad de recursos humanos necesarios cuando las tecnologías de la información no están al alcance de todos.

Este desarrollo comenzó a principios de la década de 1990, cuando se hizo un nuevo comienzo radical en la transformación de la comunidad, y cuando se reestructuró la administración, se creó una administración "invisible, sin papeles" y "las 24 horas del día" (24/7). La realización de este proyecto se lograría utilizando la tecnología de comunicación entonces moderna basada en ordenadores personales en red. (Prause, 2018, p.15)

La recepción de nuevas ideas de gestión en el sector público continuó en los años 2000 y creció con foco en la creación de valor, la digitalización y la involucración (Alford y Greve, 2017). Por su parte, estudios realizados recientemente en Suecia, muestran que sus municipios están organizados por funciones, $y$, por lo tanto, las estructuras organizacionales de estos no están alineadas con un enfoque orientado al proceso. Las responsabilidades mal definidas y mal comunicadas, son otro de los desafíos de gobernabilidad que causa ineficiencias en la gestión de procesos de los municipios (Lönn y Uppström, 2013). Diversos trabajos de investigación, como los nueve factores de Tregear y Jenkins (2007) o las tres directrices de Alford y Greve (2017), han aportado nuevos conocimientos en torno a los factores diferenciales entre el sector público y el privado, los cuales están siendo profundizados en la presente investigación, la cual ha sido motivada por la necesidad de mejorar la productividad en el sector público en la Argentina aplicando conceptos y herramientas de gestión empresarial, estableciendo las diferencias y proporcionando sugerencias para su implementación. Se busca, en resumen, hallar las diferencias clave entre la administración en entornos públicos y privados, los factores clave del éxito y la adaptación de las mejores prácticas para la mejora continua en el sector público.

\section{Revisión de literatura}

\section{Evolución de los principios de la Gestión}

Se puede definir a la Ciencia de la Gestión como un proceso de toma de decisiones y resolución de problemas llevado adelante, normalmente, por el uso de habilidades transversales y de las ciencias básicas, siempre visando la productividad, es decir, la consecución de objetivos con el uso de los recursos 
disponibles, los cuales son generalmente escasos. Cabe aquí marcar una primera diferencia, mientras que en el sector privado se tiene plena noción de que los recursos son siempre escasos, no se lo consideraría de ese modo en el sector público, donde en la mayoría de los casos parecerían considerarse ilimitados. En su libro Administración Industrial y General, en 1916, Henri Fayol define por primera vez los principios de la gestión científica. Las relaciones entre los empleados, los gerentes y las personas en general han cambiado desde ese entonces, como así también la terminología utilizada. En la década de 1950 la gestión se basaba, fundamentalmente, en el armado de un presupuesto. Se observa hoy en día, en innumerables pequeñas empresas argentinas, que el modo de gestión, en el mejor de los casos, se basa apenas en un presupuesto, no haciendo uso de herramientas que permitan una visión del largo plazo. En un trabajo de investigación desarrollado en la Argentina (Salimbeni, Barrientos, y Torres, 2018), se dice que 7 de cada 10 gerentes encuestados en pymes dicen utilizar la presupuestación como planeamiento estratégico, pero prácticamente no se utilizan técnicas ni herramientas de planificación y control como buenas prácticas. La gestión sigue entonces orientada a la organización funcional, desconociendo los principios de la Gestión por Procesos como estrategia de orientación al cliente, ya sea este interno o externo. Luego de la técnica de presupuestación, se transitó por la planificación de la producción, la planificación y dirección estratégica, hasta llegar a la gestión de la calidad y por procesos (Tabla 1).
Tabla 1

Evolución de las herramientas de gestión.

\begin{tabular}{ll}
\hline Década & \multicolumn{1}{c}{ Herramienta } \\
\hline $1920^{\prime}$ & Organización científica \\
$1950^{\prime}$ & Presupuestación \\
$1960^{\prime}$ & Planificación de la producción \\
$1970^{\prime}$ & Planificación estratégica \\
$1980^{\prime}$ & Estrategias y ventajas competitivas \\
$1990^{\prime}$ & Gestión de la calidad \\
$2000^{\prime}$ & Gestión por procesos \\
\hline
\end{tabular}

Fuente: Elaborado por el autor.

La Gestión por Procesos no es un objetivo en sí mismo sino un camino que se recorre, el cual está siendo transitado por el sector privado hace varios años ya, pero no está aún muy difundido en el sector público. Tal como señalara Tregear y Jenkins (2007) las diferencias clave entre el sector público y el privado son: (1) el interés público, (2) la responsabilidad, (3) la sensibilidad política, (4) el ecosistema de todo el gobierno, (5) la complejidad del ciclo presupuestario, (6) el intercambio de información, (7) la regulación de la sociedad, (8) la maquinaria del gobierno y (9) la cultura. Por su parte, Dixit (2002) agrega que el sector público presenta características específicas que lo hace especialmente complejo; entre estas características se destacan la multiplicidad de principales y objetivos.

\section{La Organización Funcional y por} Procesos

Es indudable que, en la actualidad, las organizaciones se desempeñan en contextos y mercados altamente competitivos y globalizados; toda organización que pretenda ser exitosa deberá obtener buenos resultados $\mathrm{y}$ 
gestionar sus actividades de manera tal de alcanzar sus metas de una manera productiva. En el ámbito público ocurre algo análogo, ya que cada vez se busca más la eficiencia y eficacia para la atención al ciudadano (Wimmer, Jannsen, y Hans, 2013). La transformación digital y la creación de redes de información han llevado a un cambio fundamental en los requisitos de información de los ciudadanos, y es por ello, por lo que los procesos administrativos en el contexto del gobierno electrónico se han tornado particularmente importantes (Wirtz y Daiser, 2018). Las organizaciones deben buscar un sistema de gestión que les permita establecer metodologías de trabajo, identificar responsabilidades, llevarlas a cabo y medir resultados con el fin de poder cumplir con las metas preestablecidas. El enfoque basado en procesos es un principio de gestión para la obtención de resultados con el objetivo de satisfacer al cliente y las demás partes interesadas. Así, cuando una organización está interesada en mejorar la forma en que presta sus servicios o elabora sus productos, el primer paso que debe llevar a cabo es analizar las diferentes actividades que la organización realiza, teniendo siempre en cuenta el enfoque en la satisfacción del cliente. Todo cliente tiene necesidades, ya sea algún problema que resolver $o$, inclusive, alguna oportunidad para explotar; la satisfacción del cliente es dar, justamente, la mejor respuesta a dichas necesidades. A partir de este enfoque ya no se observa a la organización como un conjunto de silos estancos, agrupados conforme las tareas que cada uno de ellos desempeña, o sea, por funciones, sino como una cadena de actividades que agregan valor hasta lograr el producto, servicio o resultado que el cliente espera.

La organización funcional (Figura 1) posee, como uno de sus puntos más débiles, el hecho de que cada departamento difícilmente conozca con exactitud qué es lo que otro departamento o área realiza, ni tampoco tenga muy en claro el porqué de cada una de las actividades que desempeñan, o cómo es que dichas actividades y tareas agregan valor 0 se relacionan con sus "proveedores" y "clientes". Es relativamente simple detectar actividades que no agreguen valor en este tipo de estructuras organizacionales. Se observa en muchas ocasiones que existen tareas que se desarrollan a la perfección, pero pocos pueden darse cuenta de que, en realidad, eso no es lo importante, ya que dicha tarea, perfectamente desarrollada, no está adicionando valor al producto final. Un mapeo y análisis de Flujo de Valor, conocido como análisis VSM (Value Stream Mapping), colabora estrechamente con este proceso de evaluación de actividades. En contraste a la organización funcional, la organización por procesos (Figura 2) redefine el enfoque de la gestión. La mirada está puesta ahora en el cliente, interno o externo y cada una de las actividades que se realizan para lograr ese producto, servicio o resultado que el cliente espera; se vinculan, agregan valor, conforman una cadena de actividades interrelacionadas. Todos los colaboradores dentro de la organización conocen ahora la importancia de las tareas que se desarrollan y de qué manera se está 
ayudando con el agregado de valor en cada paso. Es por ello también que la arquitectura organizacional es también de vital importancia (Rosemann y vom Brocke, 2015).

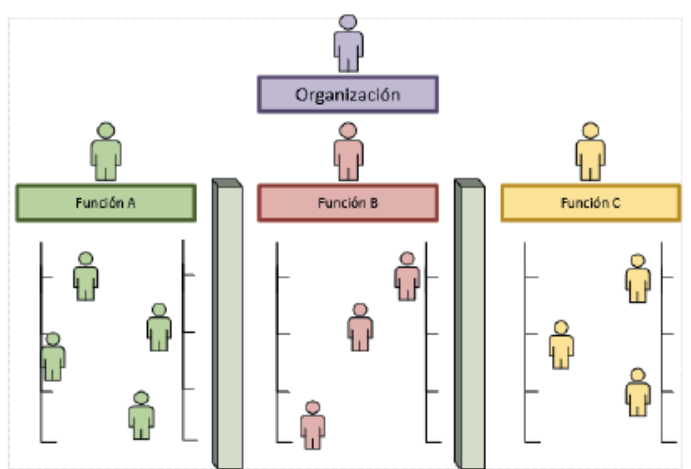

Figura 1. Organización por funciones.

Fuente: Elaborado por autor.

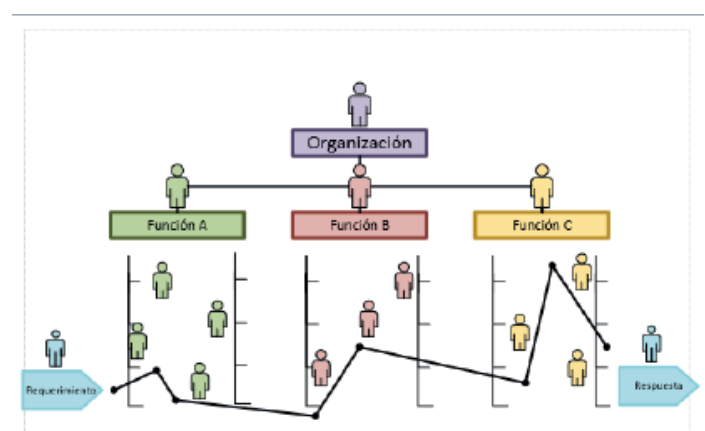

Figura 2. Organización por procesos.

Fuente: Elaborado por autor.

\section{En la búsqueda de la mejora contínua}

Gestionar es administrar, hacer buen uso de los recursos escasos para la consecución de objetivos, pero el término "Gestión", utilizado en español habitualmente como sinónimo de Administración, tiene implícito un concepto de suma importancia: la mejora continua. En general se entiende por Administrar el hacer un buen uso de lo que se dispone, mientras que Gestionar implica, además de ello, buscar la mejora continua del desempeño de la organización y siempre visando el largo plazo. Es por esto último que habitualmente se asocia a la Gestión enfocada en procesos con el ciclo de la mejora continua: planificar, implementar, medir y actuar; es un ciclo virtuoso. Este ciclo es el conocido como ciclo de Deming, de Shewhart, Ciclo de Mejora Continua o ciclo PDCA por sus siglas en inglés ${ }^{2}$ (Deming, 1989).

Para gestionar es necesario monitorear y controlar, entendiéndose por monitoreo a la medición entre los resultados que se van obteniendo y su comparación contra los objetivos preestablecidos, mientras que controlar significa, en este caso, la modificación voluntaria de las variables necesarias para reducir esa brecha entre los resultados que se van obteniendo y los objetivos preestablecidos. Para esto, como se acaba de mencionar, es necesario medir, y para poder medir se debe cuantificar, es decir, se deben establecer referencias objetivas para poder comparar los resultados obtenidos contra los resultados planificados. Esto se hace a través de métricas. Las métricas son niveles cuantificables de indicadores que se miden en un punto específico del tiempo; una métrica objetivo es el punto a alcanzar dentro de un período especificado. Un indicador es aquel que muestra el resultado de analizar una o más medidas específicas para abordar una necesidad, valor, salida, actividad o entrada. El indicador puede estar representado en una tabla o en forma gráfica. Las Normas Generales de Control Interno para el Sector Público

${ }^{2}$ Plan-Do-Check-Act. 
Nacional (Argentina), aprobadas por la Sindicatura General de la Nación (SIGEN) en el año 2014 caracterizan al control interno como un proceso integrado a los procesos básicos de planificación, ejecución y supervisión, y no a un conjunto de mecanismos burocráticos añadidos a los mismos (Ivanega, 2016). Al respecto se destaca que el monitoreo y control de procesos no debe entorpecer o burocratizar la gestión.

\section{La transición hacia la Gestión por} Procesos

La mayoría de las reconversiones actuales se basan en el desarrollo de pequeños proyectos de mejora $o$ resolución de problemas, los cuales normalmente no revisten demasiada complejidad. Existen otros proyectos que son lo suficientemente abarcativos como para ser realmente transformadores, ofreciendo cambios fundamentales en la forma en que se ve la operación y la manera en que se realiza el trabajo, pero con mayor frecuencia se realizan cambios de reglas, operaciones, políticas y procedimientos que con el tiempo se transforman en leyes no escritas y no aprobadas que modifican la forma en que funciona la organización. Estos cambios causan interrupciones constantes, perjudican la productividad y crean un conjunto de regulaciones involuntarias en torno al trabajo real. Esto ocasiona problemas, siendo el mayor de ellos la acumulación y aumento de los desvíos, bastante graves en algunas oportunidades. Esto ocurre en las entradas (proveedores) como en las salidas (clientes) de cada proceso. Este agravamiento progresivo sucede bajo la visión organizacional y los límites que impone al observar el cambio y su impacto. Una de las razones clave para progresar hacia una perspectiva de proceso y crear un enfoque de Gestión por Procesos es controlar dichos cambios y mejorar la calidad y el desempeño de la organización. La Gestión por Procesos no se debe observar en términos de una estructura de organización tradicional; está virtualmente separada y no se alinea con la organización. En realidad, el proceso es transversal a la organización, atraviesa múltiples áreas, o inclusive diferentes organizaciones y cada una agrega algún componente o parte del producto, servicio o resultado final. Dichos procesos no necesariamente deben estar limitados a un área o incluso a una sola empresa, pudiendo representar el intercambio de actividades entre proveedores y clientes externos.

\section{Marco de referencia de procesos}

Es fundamental, para toda organización, un modelo de procesos corporativo. La mayoría de las organizaciones y empresas se benefician enormemente al utilizar un modelo de referencia de procesos como un punto de partida para su clasificación y organización. Los marcos de referencia de procesos pueden ser, por ejemplo:

- Aplicables, generalmente, a diferentes tipos de empresas $u$ organizaciones, como el modelo de clasificación de procesos APQC (American Productivity \& Quality Center), o el modelo de referencia operativa de cadena de valor, VRM. 


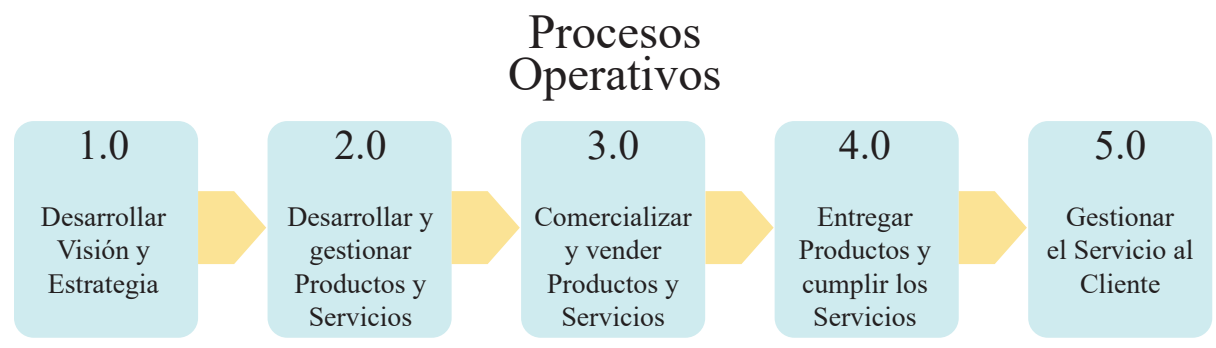

Figura 3. Marco de Referencia de Procesos. Fuente: APQC (2014).

- Específicos para una industria (modelo de referencia de operaciones de cadena de suministro).

- Específicos para un área de proceso (Biblioteca de Infraestructura de Tecnología de la Información).

- Específicos para la tecnología (SAPTM).

Existen otros marcos de referencia, como el PFC (Process Classification Framework), definido por la APQC (2014) que es la principal autoridad mundial en evaluación comparativa, mejores prácticas, mejora de procesos y desempeño y gestión del conocimiento. Como organización sin fines de lucro la convierte en un elemento diferenciador en el mercado; es una asociación internacional de benchmarking que ha colaborado con 80 organizaciones en el desarrollo de un marco de referencia para la evaluación de procesos (Figura 3).

El Marco de Clasificación de Procesos puede ser utilizado por las organizaciones como punto de partida para un Modelo de Proceso Empresarial. El Marco de Clasificación de Procesos de APQC pretende servir como un modelo empresarial de alto nivel e independiente de la industria que permite a las organizaciones ver sus actividades desde un punto de vista de proceso entre industrias (Figura 4).

Gestión y Soporte de Procesos

6.0 Desarrollar y gestionar Capital Humano

7.0 Gestionar Tecnologías de la Información

8.0 Gestionar Recursos Financieros

9.0 Adquirir, construir y gestionar Bienes

10.0 Gestionar la Salud y Seguridad Ambiental

11.0 Gestionar Relaciones Externas

12.0 Gestionar Conocimientos, Mejoras y Cambios

Figura 4. Marco de Clasificación de Procesos. Fuente: APQC (2014).

\section{La Gestión por Procesos en el Sector} Público

Como tantas otras herramientas de gestión, la Gestión por Procesos nació y se desarrolló en el sector privado, y la mayor parte de la literatura sobre el tema se desarrolla en ese ámbito. Los ambientes más fuertemente competitivos hicieron que las compañías comenzaran a tomar mucho más en cuenta al cliente y fue así como el enfoque se orientó 
definitivamente hacia él.

Existe un interés creciente en la gestión basada en procesos. BPM está ganando aceptación como una filosofía y práctica de gestión efectiva y holística. Gran parte de la literatura de gestión de procesos se centra en las organizaciones del Sector Privado con suposiciones inherentes de resultados tangibles, basados en los beneficios y grupos de clientes bien definidos. (Tregear y Jenkins, 2007, p.1)

La virtud de trabajar por procesos permitió introducir técnicas $\mathrm{y}$ herramientas con el fin de mejorar la calidad y reducir los desperdicios. Así, por ejemplo, comenzaron a utilizarse las prácticas Lean-Six Sigma, ya no sólo en las industrias de transformación sino también en las de servicios. Es allí cuando comienzan a difundirse las mejores prácticas, no sólo a servicios en el sector privado, sino en la Administración Pública también, siempre buscando satisfacer más eficientemente las necesidades de los ciudadanos. Si bien la productividad es importante desde el punto de vista de la Administración Pública, probablemente el usuario final del servicio, el ciudadano, esté más interesado en los resultados más que en la eficiencia (Girard y Minvielle, 2002).

Como ya se ha mencionado, el Sector Público posee características distintivas respecto del privado. Ciertos factores o variables son los que se comenzaron a analizar con el fin de adaptar el BPM, nacido para empresas privadas, a la Gestión por Procesos en el sector público; a partir de allí es que se acuñó un nuevo término: GPM. En un estudio de la Universidad de Pernambuco realizado por Valenca, Frota Alves, Lemos Santana, Pereira de Oliveira, y Monteiro Santos (2013) encontraron que: (1) un patrocinio fuerte, (2) una capacitación adecuada en BPM y (3) la disponibilidad de personal interno con experiencia en BPM, son los factores clave de éxito para estas iniciativas.

La Secretaría de Gestión Pública del Perú (2013) indica tener en cuenta las siguientes condiciones previas a la implementación de la gestión por procesos: (1) Asegurar el apoyo y compromiso de la Alta Dirección, (2) contar con objetivos estratégicos claramente establecidos, (3) asegurar los recursos, y (4) fortalecer el órgano responsable de la implementación de la Gestión por Procesos.

Se ha estado trabajando en varios países, fundamentalmente durante esta última década, en entender y proponer adaptaciones del BPM para llevarlo al GPM. Así es que, en ciertos casos, se lo incorpora como parte de políticas públicas. En la gestión pública peruana, el enfoque de Gestión por Procesos es parte del Tercer Eje de la Política de Modernización Pública, cuyo fin es construir una gestión moderna y orientada a resultados, al servicio del ciudadano (Martínez Escobar, 2015).

Si bien ciertos problemas son comunes y habituales en la gestión de empresas privadas, algunos de ellos se agudizan en ámbitos públicos, como por ejemplo los 
inconvenientes en la comunicación o la identificación de roles y responsables de las partes interesadas. Un estudio realizado en Suecia en 2013 así lo confirma: las responsabilidades mal definidas y comunicadas son otro desafío de gobernabilidad que causa ineficiencias en la gestión de procesos de los municipios (Lönn y Uppström, 2013). Otra de las diferencias entre lo público y privado es que existen recursos de entrada fuera del control en los organismos públicos, los cuales afectan al resultado de las agencias (Coleman et al, 1966).

\section{Metodología de investigación}

Se detalla a continuación la metodología, tanto para el relevamiento bibliográfico como para el trabajo en campo. El relevamiento de datos para la producción del estado de arte se ha desarrollado en el período mayo de 2018 a abril de 2019. El análisis cuantitativo finalizará en agosto de 2020. Se ha decidido por un método mixto, debido a que este ofrece varias bondades, tales como: (1) Lograr una perspectiva más amplia y profunda del fenómeno (2): formular el planteamiento del problema con mayor claridad, (3) producir datos variados y de mejor calidad mediante la multiplicidad de observaciones, (4) potenciar la creatividad teórica por medio de suficientes procedimientos críticos de valoración, (5) efectuar indagaciones más dinámicas, (6) apoyar con mayor solidez las inferencias científicas y (7) permitir una mejor "exploración y explotación". (Hernández Sampieri, Fernández Collado, y Baptista Lucio, 2010, p.549-550)
El enfoque es cuali-cuantitativo y desde la perspectiva de las Ciencias Económicas y Empresariales. El alcance de la investigación es exploratoria y descriptiva, con observación participante y cuestionario estructurado, presenciado en los propios escenarios donde transcurre la observación. Es un diseño no experimental, transaccional y de tipo exploratorio.

Respecto del marco teórico, la metodología empleada fue con método de mapeo y mapa conceptual con confección de fichas de lectura. La revisión bibliográfica constó de seis pasos: (1) En bases de datos académicas con cadena de búsqueda a través de la combinación del operador "o" entre las palabras clave se recogieron las referencias que cumplían con los siguientes criterios: (a) fueron publicados en línea; (b) contenían, al menos, uno de los términos de búsqueda en el resumen, título y / o palabras clave y (c) se publicaron en actas de congresos, artículos, revistas, series de libros, y libros. (2) Se eliminaron los duplicados. (3) Se descartaron los que no tenían a disposición textos completos. (4) $\mathrm{Se}$ excluyeron los documentos que definieron la Gestión por Procesos fuera del alcance de este trabajo de investigación. (5) Se clasificaron conforme las preguntas de investigación. (6) Se revisaron los documentos colectados y recopilaron los datos correspondientes de interés para las preguntas de investigación.

La recolección, evaluación y análisis de datos se realizó en las etapas 5 y 6 conforme las siguientes preguntas de 
investigación (Q) e hipótesis (H): (Q1) Considerando que la mayoría de las herramientas y conceptos de gestión estratégica se desarrollaron en el Sector privado ¿es válido aplicarlos al Sector Público? (Q2) ¿Cuál es la importancia de la Gestión por Procesos en el Sector Público? (Q3) Las diferencias clave halladas en otros países entre el Sector Público y el Sector privado ¿son aplicables a la Argentina? (Q4) ¿De qué forma puede ser implementado el concepto de Gestión por Procesos en la Administración Pública? Las hipótesis son: (H1) La aplicación del BPM al Sector Público, conocido también como GPM, puede mejorar notablemente su productividad. (H2) La Administración Pública no tiene aún asimilado ni aplicado el concepto GPM. (H3) Las diferencias y dificultades en la aplicación de la Gestión por Procesos en el Sector Público son muy similares en todos los países. (H4) Una mayor capacitación y formación de los cuadros de gobierno facilitarían la implementación de la Gestión por Procesos.

En relación al trabajo en campo, las unidades de análisis y respuesta fueron, para la etapa cualitativa, 2 conjuntos de 5 organizaciones, 5 privadas y 5 públicas, como unidad de respuesta y de análisis. Para la etapa cuantitativa se tomó como unidad para el análisis comparativo a 50 interlocutores del sector privado y a 50 del sector público.

La técnica de recolección de datos para la etapa cualitativa ha sido entrevistas en profundidad; "lo que sucede y el modo en que otras personas lo perciben" (Taylor y Bogdan, 1987, p.103). Para la etapa cuantitativa, la técnica utilizada fue la encuesta. Este tipo de entrevista estandarizada se lleva a cabo por medio de un cuestionario estructurado, lo que permite comparar distintas respuestas ante la misma pregunta y cuantificar cada uno de los resultados obtenidos (García Ferrando, 1996, p.178). Por medio de esta técnica se busca verificar las hipótesis. Respecto de las muestras, para la etapa cualitativa se recurrió a la estrategia del muestreo teórico para seleccionar las organizaciones e individuos a entrevistar. En la etapa cuantitativa, la encuesta se administró a un grupo de personas denominado: la muestra, con la pretensión de identificar tendencias en actitudes, opiniones, comportamientos o características de un grupo más grande de individuos denominados: la población. Para la obtención de los contactos se siguió la siguiente secuencia: se envió la encuesta a 100 contactos, 50 del sector privado y 50 del sector público. Se espera obtener finalmente, al menos, 50 casos (25 del Sector privado y 25 del Sector Público) que respondan en forma completa y válida la encuesta.

Para el procesamiento y análisis de datos durante la etapa cualitativa se realizó un análisis comparativo y por factores. Para la etapa cuantitativa se realizaron las encuestas en línea a través de la plataforma "e-encuestaTM", la cual generó una matriz de datos de las respuestas. El diseño metodológico, la elaboración de los instrumentos de recolección de datos, la realización del trabajo en campo, el procesamiento y el 
análisis estuvieron a cargo del equipo de investigación de la Universidad del Salvador. Se contó con la colaboración de alumnos practicantes para lograr acceder a un número mayor de encuestas. Las entrevistas en profundidad se realizaron en el AMBA (Área Metropolitana Buenos Aires) y las encuestas en línea se dirigieron a organizaciones privadas $\mathrm{y}$ públicas en toda la Argentina.

\section{Resultados}

Durante la etapa cualitativa de la investigación se comienza a fortalecer la hipótesis de que las debilidades en Planificación y Gestión por Procesos son aún más marcadas en el sector público debido a la mayor rigidez de sus políticas y normativas, como así también la preponderancia de decisiones políticas por sobre las necesidades del cliente / ciudadano. La Inteligencia de Procesos y las nuevas herramientas de simulación pueden colaborar aún más en la Gestión por Procesos en el Sector Público; esto es debido a que no es necesario experimentar en campo los procesos mejorados, ya que se los puede simular evaluando tiempos y costos a través del uso de software específico y con un nivel de conocimiento en herramientas informáticas no muy profundo. Como Mendling y Weber (2018) ya habían enunciado, se corrobora que se requiere de la ciencia y las tecnologías de la información, la ingeniería de software y los sistemas distribuidos para mejorar las vías de información al cliente/ciudadano.

Durante las entrevistas en profundidad se han evaluado el grado de validez de las nueve diferencias clave entre el sector público y el sector privado (Tregear y Jenkins, 2007), a saber: (1) Interés público, (2) responsabilidad, (3) sensibilidad política, (4) ecosistema de todo el gobierno, (5) complejidad del ciclo presupuestario, (6) intercambio de información, (7) regulación de la sociedad, (8) maquinaria de cambios gubernamentales y (9) cultura y los factores clave de éxito para la implementación de estas iniciativas. Se han explorado también los factores clave de éxito para su implementación (Tabla 2). Todo ello será posible cuantificarlo durante la próxima fase del estudio.

Tabla 2.

Diferencias y factores clave.

\begin{tabular}{ll}
\hline $\begin{array}{l}\text { Tregear y Jenkins (2007) } \\
\text { Diferencias clave entre el } \\
\text { sector público y el sector } \\
\text { privado }\end{array}$ & $\begin{array}{l}\text { Factores clave de éxito } \\
\text { para la implementación } \\
\text { de GPM }\end{array}$ \\
\hline $\begin{array}{l}\text { Interés público } \\
\text { Responsabilidad }\end{array}$ & $\begin{array}{l}\text { Patrocinio fuerte } \\
\text { Capacitación adecuada } \\
\text { en BPM } \\
\text { Disponibilidad de personal } \\
\text { interno con experiencia en }\end{array}$ \\
Sensibilidad política & BPM \\
$\begin{array}{l}\text { Ecosistema de todo el } \\
\text { gobierno }\end{array}$ & \\
$\begin{array}{l}\text { Complejidad del ciclo } \\
\text { presupuestario }\end{array}$ & \\
$\begin{array}{l}\text { Intercambio de información } \\
\text { Regulación de la sociedad } \\
\text { Maquinaria del gobierno } \\
\text { Cultura }\end{array}$ & \\
\hline
\end{tabular}

Fuente: Elaborado por el autor.

Como ya se ha mencionado, si bien existen numerosos estudios a nivel local respecto de los procesos de negocios BPM, la gran mayoría de ellos están enfocados al sector privado y casi todos relacionados con desarrollos de software. Este estudio aporta una nueva perspectiva desde la gestión pública, sin estar 
relacionado intencionalmente a las herramientas informáticas, sino a los procesos en sí mismos. El presente trabajo de investigación programado para ser finalizado en agosto de 2020 está, a la fecha, en un $50 \%$ de avance. No obstante ello, se está en condiciones de compartir algunos incipientes hallazgos.

A partir de las entrevistas en profundidad en las cinco empresas privadas y en los cinco sectores públicos se extraen los siguientes hallazgos:

Al momento de analizar la Gestión por Procesos en la Administración Pública resulta clave comprender que se tiene un corrimiento con respecto a los objetivos organizacionales. Las organizaciones públicas se gestionan según el ritmo de la política, lo cual no sólo ocurre en los cargos de alta Dirección, sino también en los mandos medios. La estructura pública se mueve conforme los tiempos políticos. Esto coincide con lo postulado por Pedraja Chaparro, Jiménez y Jiménez (2005) donde especifican que muchos de los efectos de las actuaciones públicas se manifiestan en el mediano y largo plazo. Si el sistema se aplicara en un horizonte temporal muy limitado podrían plantearse problemas de atribución de resultados y los agentes tendrían incentivos para centrar sus esfuerzos en objetivos de corto plazo en detrimento de aquellos objetivos de mediano y largo plazo. Este problema se conoce como "miopía temporal" y puede verse agravado por el hecho de que muchos de los gestores ocupan sus cargos durante un periodo de tiempo relativamente corto.
Varios organismos públicos en el país han optado por avanzar en el desarrollo de la Gestión por Procesos, aunque los resultados suelen ser dispares y en ocasiones el único fin es tan solo publicitar que se aplican Prácticas de Nivel Mundial, sin comprenderlo como una política o herramienta clave que logre optimizar los recursos. Sin embargo, otros lo consideran como una gran oportunidad de gestionar los organismos públicos de manera más eficiente, sabiendo que ello implica beneficios para el ciudadano a mediano y largo plazo.

Uno de los Gerentes de la Dirección General de Relocalización y Gestión Integral de Edificios de Gobierno de la Ciudad Autónoma de Buenos Aires (DGRGIEG) dice que en su organismo están presentes todas las características de una organización por funciones; los equipos no tienen total claridad sobre las actividades que los otros colaboradores realizan, ni comprenden, muchas veces, la importancia de su trabajo; sin embargo, agrega, el grado de organización es elevado y tan solo se requiere de la comprensión de que cada uno de ellos es proveedor y cliente de otro proceso cuyo cliente final es el ciudadano de la Ciudad de Buenos Aires. Una apreciación adicional, de parte de este funcionario, fue que la Gestión por Procesos en la Ciudad debe estar igualmente orientada a la calidad, solo que la calidad ya no es percibida por un cliente consumidor de un producto o servicio, sino por un ciudadano que es "compulsivamente" cliente del gobierno; los indicadores clave de desempeño deben estar claramente ligados a los procesos que 
realmente potencien dicha satisfacción. En el caso de un gobierno municipal, el objetivo debe ser lograr que las personas puedan vivir mejor en su ciudad.

Luego de comprender que los tiempos políticos son el gran obstáculo, existe otro, aunque existe forma de superarlo: la resistencia al cambio de los recursos humanos que componen las organizaciones, y este no es exclusivo de la actividad pública. Dicho funcionario complementa también que es habitual que, debido a las características de las contrataciones en organismos públicos y leyes que lo regulan, el personal suele estar estancado y amarrado a su trabajo, por lo tanto, se convierte en un total opositor al cambio. En organizaciones pequeñas la gestión es más simple, pero no así en organismos públicos del tamaño de compañías multinacionales; se habla muchas veces de alrededor de 30.000 empleados que deben ser formados y capacitados. Los desafíos están planteados y sin dudas pueden ser superados; solamente queda lograr que todos los que componen el Estado entiendan que los beneficios superan ampliamente los costos asociados a estas transformaciones.

Se ha realizado un ejercicio comparativo de comunicación de procedimientos en una empresa mediana y en un área del CESBA, las cuales en cuanto a cantidad de empleados son comparables, y utilizando las herramientas de comunicación propias disponibles. Los resultados se dieron a favor del sector privado, $y$, aunque no se pueden valorar como definitivos, la evaluación actual es que en la oficina de gobierno la información fluye con mayor lentitud e inexactitud, pudiendo haber sido causado por la mayor burocracia, menor uso de tecnología y menor conocimiento en la materia.

Por su parte, y a partir de observaciones y entrevistas en diferentes áreas gobierno, se pudo observar que los representantes de los organismos públicos confunden muchas veces el organismo en sí mismo con su política partidaria y hasta personal. Confundir gestión pública con política lleva generalmente a que los roles de las personas que ingresan temporalmente por períodos políticos se solapen con las tareas específicas que personal de planta permanente venía desarrollando, tornándose totalmente improductivo. En el sector público de la Argentina, las personas en planta permanente llevan un largo tiempo trabajando en el mismo puesto, lo cual genera indefectiblemente acostumbramiento, baja proactividad y una dedicación orientada sólo a las actividades básicas que le corresponden. Por su parte, el personal contratado o temporal, el cual cumple ciclos de 2 ó 4 años, coincidiendo exactamente con los períodos electorales en la Argentina, está más motivado y orientado a resultados, pero al no haber procesos y procedimientos formales dentro del organismo público no conocen qué actividades deben realizar y en muchas ocasiones no se tiene en cuenta si la persona posee la formación y las habilidades para desempeñar dichas actividades. Es bastante frecuente encontrar que tampoco se conozcan claramente los roles y responsabilidades de cada puesto, y a esto se le suman 
también los tiempos ociosos, los cuales normalmente no se toman en consideración.

En contraste con lo anterior, en el sector privado en la Argentina, en general, las tareas se encuentran descritas por cada puesto de trabajo y la persona conoce claramente cuáles son sus responsabilidades, como así también los objetivos preestablecidos por su empresa, sabiendo que deben alcanzarlos y que serán evaluados conforme la consecución de estos, en cuyos casos existen premios y penalidades. En 4 de las 5 empresas privadas consultadas, se ha encontrado una descripción de puestos, roles $\mathrm{y}$ responsabilidades completos, no siendo así en las 5 áreas analizadas pertenecientes al sector público.

Se han detectado otros factores de diferenciación entre el sector público y el privado. Durante la entrevista a un Gerente de Área de la DGRGIEG manifestó: "ningún organismo del Estado con los que he trabajado tiene un plan de contingencia para el caso en que las personas roten o por algún motivo dejen el puesto". Él mismo agrega que, en el sector privado, se intenta desarrollar procedimientos y descripciones de tareas para que, en el caso que alguien se ausente, otro pueda ocupar dicho rol rápidamente. En cambio, en gobierno, dice el mismo funcionario, se tienen empleados con muchos años en el puesto sin documentación formal asociada, por lo cual cuando alguno de ellos cambia de sector o se retira, el que ingresa no tiene suficientes documentos que solventen sus tareas; agrega también que podría mejorarse dicha situación si cada área de gobierno contara con procesos y procedimientos estandarizados y documentados; si además de ello estos fueran cumplidos a consciencia, se les daría un mejor asesoramiento al ciudadano y los problemas se resolverían más rápidamente. Este efecto es claro, la pregunta al momento sigue siendo si todos los funcionarios son conscientes de las causas que lo producen.

Tomando como referencia las 8 gerencias donde se han realizado las entrevistas en profundidad, se ha hallado también que las organizaciones públicas, en general, no cuentan con suficientes especialistas en proyectos y procesos, por lo cual, muchas veces, no llegan a cumplir los objetivos anuales y no tienen planes de contingencias para encarar situaciones críticas.

\section{Conclusiones}

Luego de esta primera fase, y tomando como referencia a los factores citados por Tregear y Jenkins (2007), se puede decir que: (1) En relación al Interés Público: no se evidencia una toma de consciencia de la importancia de la eficiencia y eficacia en la gestión pública; se continúa con la idea, en general, de que los recursos por ser públicos tienen costo cero. (2) Responsabilidad: no se evidencia en este caso falta de responsabilidad por parte de los funcionarios, sino cierta falta de conocimientos de técnicas y herramientas de las ciencias de la gestión. (3) Sensibilidad Política: el exceso de la orientación de las actividades hacia la 
inmediatez de los resultados hace perder la perspectiva del largo plazo. La política les impone generalmente resultados acotados a períodos breves; se evidencia la denominada "miopía temporal". (4) Ecosistema de Gobierno: esperamos la fase cuantitativa para tener una conclusión válida al respecto. (5) Complejidad del Ciclo Presupuestario: las aprobaciones, modificaciones $\mathrm{y}$ actualizaciones presupuestarias, conllevan un proceso lento y complejo, lo cual quita cierta flexibilidad a la gestión. Este punto es bien diferente respecto del sector privado. (6) Intercambio de Información: la debilidad de infraestructura tecnológica, aunque mejorada notablemente durante estos últimos años, y la carencia de habilidades blandas, impactan negativamente en la comunicación. (7) Regulación de la Sociedad, (8) Maquinaria de Cambios Gubernamentales y (9) Cultura, son todos aspectos bien diferentes de aquellos del sector privado limitando también las correcciones, en tiempo y forma, cuando se apartan del plan, del camino que los conduciría a la consecución de los objetivos preestablecidos.

En respuesta a las preguntas de investigación se puede concluir al momento que: (1) aunque la mayoría de las herramientas y conceptos de gestión estratégica se desarrollaron en el sector privado, es válido aplicarlos al sector público, siempre tomando en consideración los límites naturales de los procesos y ciertas variables externas no tan fácilmente controlables en la gestión pública. (2) La gestión por procesos en la Administración Pública es una herramienta que permite trabajar en función de las necesidades de los ciudadanos, sin descuidar los recursos insumidos para lograrlo; trabajar por procesos permite la definición de indicadores de gestión, los cuales colaboran con un círculo virtuoso y, por ende, de mejora continua. (3) Las diferencias clave halladas en otros países entre el sector público y el sector privado son aplicables a la Argentina, e.g (a) patrocinio fuerte, (b) capacitación adecuada y (c) personal interno con experiencia. (4) La mayor burocracia y "silos" en áreas gubernamentales hace que se deba aplicar una estrategia de transición moderada acompañada por una fuerte capacitación para que realmente la Gestión por Procesos pueda ir incorporándose a la Administración Pública.

Se espera para agosto de 2020 haber finalizado el estudio cuantitativo detallado de los factores de diferenciación entre la Gestión por Procesos en el sector público y privado de la Argentina, así como también una serie de técnicas y herramientas recomendadas y adecuadas que sirvan como una guía para la aplicación de la Gestión por Procesos en el sector público.

\section{Referencias}

Alford, J. (2001). The Implications of "Publicness" for Strategic Management Theory, in G Johnson \& K Scholes (eds), Exploring Public Sector Strategy, Prentice-Hall, London.

Alford, J. y Greve, C. (2017). Strategy in the Public and Private Sectors: Similarities, Differences and Changes. Administrative 
Sciences, 7(4), 35. doi: 10.3390 /admsci7040035

APQC. (2014). Process Classification Framework, version 6.1.1. Recuperado de www. Apqc.org

Bower, J.L., Bartlett, C.A., Christensen, C.R., Pearson, A.E., Andrews, K.R. (1991). Business policy: text and cases. $7^{\text {th }} \mathrm{ed}$. Homewood, IL: Irwin.

Coleman, J.S., Campbell, E.Q., Hobson, C.J., MacPartland, J., Mood, A.M., Weinfeld, F.D., y York, R.L. (1966). Equality of Education Opportunity, Washington, DC: US. GPO.

Deming, W.E. (1989). Calidad, productividad y competitividad. La salida de la crisis. Madrid, España: Díaz de Santos.

Dixit, A. (2002). Incentives and organizations in the Public Sector. An Interpretive Review. Journal of Human Resources, 37(4), 696-727.

García Ferrando, M. (1996). El análisis de la realidad social. Métodos y técnicas de investigación. 3era edición. Madrid: ed. Alianza.

Girard, J.F. y Minvielle, E. (2002). Measuring up: lessons and potential. En Measuring up: improving Health System Performance in OECD Countries (pp. 337-347). París, Francia: OECD.

Hernández Sampieri, H. Fernández Collado, C. y Baptista Lucio, M. (2010). Metodología de la investigación. México: McGraw-Hill Interamericana.

Ivanega, M. M. (2016). Gestión Pública y Control Interno. Asociación de Docentes Facultad de Derecho y Ciencias Sociales Universidad de Buenos Aires.

Lönn, C.M. y Uppström, E. (2013). Process Management Challenges in Swedish
Public Sector: A Bottom Up Initiative. In: Wimmer M.A., Janssen M., Scholl H.J. (eds) Electronic Government. EGOV 2013. Lecture Notes in Computer Science, 8074, 212-223.

Marchán, C. y Oviedo, M. (2011). Modelo de Organización y Gestión por Procesos en la Administración Pública del Ecuador. $1^{a}$. Ed. Quito: Editorial IAEN.

Martínez Escobar, A. (2015). Una aproximación metodológica a la aplicación de técnicas de BPM para la mejora de los procesos de negocio en la Administración Pública (tesis de postgrado), Universidad de Granada, Granada, España.

Mendling, J. \& Weber, I. (2018). Blockchains for Business Process Management Challenges and Opportunities. $A C M$ Transactions on Management Information Systems, 9(1), 1-16.

Pedraja Chaparro, F., Jiménez, J. y Jiménez, M. (2005). Los indicadores de gestión en el sector público. Revista de Economía y Estadística, Cuarta Época, 43(2), 109-129.

Prause, G. (2018). e-Government als Standortfaktor. Workshop in the frame of the 11th Wismar Business Informatics Day 2018 WIWITA, Wismar, Germany.

Rosemann, M. y vom Brocke, J. (2015). The six core elements of business process management. In Handbook on Business Process Management 1 (pp. 105-122). Berlin, Germany: Springer Berlin Heidelberg.

Salimbeni, S., Barrientos, S., y Torres, E. (2018). Planeación Estratégica y Evaluación de Riesgos Empresariales. https://doi.org/10.13140/RG.2.2.27786.1 6324

Secretaría de Gestión Pública de la Presidencia del Consejo de Ministros de Gobierno del 
Perú. (2013). Metodología para la implementación de la Gestión por Procesos en las entidades de la Administración Pública en el marco del D.S. $\quad N^{\circ}$ 004-2013-PCM - Política Nacional de Modernización de la Gestión Pública. Recuperado de:

http://sgp.pcm.gob.pe/wp-content/upload s/2015/03/Metodologia_de_GxP.pdf

Taylor, S. J. \& Bogdan R. (1987). Introducción a los métodos cualitativos de investigación: la búsqueda de significados. Barcelona: Paidós.

Tregear, R. \& Jenkins, T. (2007). Government Process Management: A review of key differences between the public and private Sectors and their influence on the achievement of public Sector process management. Recuperado de:

https://www.bptrends.com/bpt/wp-conten t/publicationfiles/10-07-ART-Govt.Proces sMgt.-Tregear\%20and\%20Jenkins-ph.pdf

Valenca, G., Frota Alves, C., Lemos Santana, A., Pereira de Oliveira, J. y Monteiro Santos, H. (2013). Understanding The Adoption Of BPM Governance In Brazilian Public Sector. ECIS Proceedings, Association for Information Systems.

Wimmer, M., Janssen, M. y Hans, J. (2013). Electronic Government 12th IFIP WG 8.5. In International Conference, EGOV 2013, Koblenz, Germany.

Wirtz, B.W. y Daiser, P. (2018). E-Government. In: Voigt R. (eds), Handbuch Staat (pp.981-995). Netphen, Germany: Springer VS, Wiesbaden. 